BULL. AUSTRAL. MATH. SOC.

VOL. $20(1979), 385-395$.

\title{
On the stability of \\ barrelled topologies, I
}

\section{W.J. Robertson and F.E. Yeomans}

\begin{abstract}
This note investigates, for locally convex topological vector spaces, the question of how far the property of being barrelled is stable under small increase in the size of the dual space. If the dual $F$ of a barrelled space $E$ is enlarged by a finite dimensional vector space $M$, then $E$ remains barrelled under the new Mackey topology $\tau(E, F+M)$. We discuss what happens when $M$ has countable dimension.
\end{abstract}

1. Since the property of being barrelled carries over to all products, sums and quotients, the study of its permanence has been concentrated mainly upon the question of inheritance by some particular types of vector subspace. Dieudonné showed in 1952 [4] that, in a barrelled space, any hyperplane, and so any vector subspace of finite codimension, is barrelled. It was not until 1971 that this was finally extended to:

THEOREM A. A vector subspace of countable codimension in a barrelled space is barrezled.

This was proved by Saxon and Levin [7] and by Valdivia [9]; the same result for a metrisable space had been established by Amemiya and Kōmura three years previously [1].

Here, instead of varying the size of the space by a small amount, we perturb its dual, by adding to it a finite or countable number of

Received 3 April 1979. The authors are grateful to Professor H.G. Garnir for drawing the paper [3] to their attention. An error in this paper was pointed out to them by $\mathrm{Dr}$ Ian Tweddle (see p. 387, footnote), and they would like to thank Dr Tweddle for helpful discussions and for communication of further results. 
dimensions.

We suppose throughout that the spaces considered are all locally convex Hausdorff spaces over the real or complex field. To avoid repetition, we shall always suppose that $E$ is a barrelled space with dual $F$; then its topology is the Mackey topology $\tau(E, F)$. The algebraic dual of $E$ is denoted by $E^{*}$, the value of the linear form $f$ in $E^{*}$ at $x$ in $E$ being written as $f(x)$. Otherwise the notation and terminology are those of [6]. We use "countable" to mean "countably infinite".

2. We deal first with the finite dimensional case.

LEMMA. Let $\tau(E, F)$ be barrezzed and $f_{0} \in E^{*} \backslash F$. Then $\tau\left(E, E+\operatorname{span} f_{0}\right)$ is barrezzed.

This lemma is stated, in slightly different form, by De Wilde and Schmets ([3], 2a and 2b), and proved by using the theorem of Dieudonné referred to above. For the sake of completeness, we give a brief indication of a direct proof, based on Dieudonné's method.

Proof. If $B$ is a bounded subset of $F+\operatorname{span} f_{0}$ and $g \in B$, then $g=f+\lambda f_{0}$ with $f \in F$, so that $B \subseteq A+\Lambda f_{0}$, say. If $\Lambda$ is not bounded there exist $g_{n} \in B, f_{n} \in A, \lambda_{n} \in \Lambda$, with $g_{n}=f_{n}+\lambda_{n} f_{0}$ and $\left|\lambda_{n}\right| \rightarrow \infty$. It follows that $\lambda_{n}^{-1} f_{n} \rightarrow-f_{0}$ in $\sigma\left(F+\operatorname{span} f_{0}, E\right)$ and then that $f_{0} \in E$, since $\tau(E, E)$ is barrelled. Thus $\Lambda$, and so also $A$, are bounded; then $B$ lies in the sum of two compact sets.

From the lemma we have immediately:

THEOREM 1. If $\tau(E, F)$ is barrezled and if $N E^{*}$ is finite dimensional, then $\tau(E, F+N)$ is barrelled.

3. There is no parallel to Theorem 1 in which the dual is decreased. Even if $H$ is a hyperplane in $F$ (such that $(E, H)$ is a dual pair), $\tau(E, H)$ may or may not be barrelled, as the following simple examples show.

(a) Let $E$ be barrelled under a topology $\tau(E, G)$ with $G \neq E^{*}$, let $f_{0} \in E^{*} \backslash G$, and let $F=G+\operatorname{span} f_{0}$. Then by the lemra $\tau(E, F)$ is barrelled. Let $H=G$; then $\tau(E, H)$ is barrelled. 
(b) Let $E$ be a Banach space and $H$ a dense hyperplane in $F$. Then $\tau(E, H)$ is strictly coarser than the fully complete topology $\tau(E, F)$ and so by the closed graph theorem can not be barrelled.

4. In all that follows, we shall use $H$ to denote a vector subspace of $E^{*}$, of countable dimension, with iM $\cap E=\{0\}$. Write $M=\bigcup_{n=1}^{\infty} M_{n}$, with $\left(M_{n}\right)$ increasing and each $M_{n}$ n-dimensional; then Theorem 1 shows that there is on $E$ a sequence of barrelled topologies $\tau\left(E, F+M_{n}\right)$, each finer than the preceding. The first natural question to ask is whether, by analogy with Theorem $A$, there is a theorem asserting that the Mackey topology $\tau(E, F+M)$ is always barrelled. But it is easy to prove:

THEOREM 2. If $E$ has an infinite dimensional bounded set then there exists $M$ such that $\tau(E, F+M)$ is not barrelied.

Proof. Let $A=\left\{e_{1}, e_{2}, \ldots\right\}$ be a bounded linearly independent infinite set and extend to form a Hamel basis for $E$. For each $n$ define a linear form $f_{n}$ on $E$ by putting $f_{n}\left(e_{n}\right)=n$ and $f_{n}$ zero on the remaining basis elements. Then the set $B=\left\{f_{1}, f_{2}, \ldots\right\}$ is linearly independent and $\sigma\left(E^{*}, E\right)$-bounded. Let $G=F+\operatorname{span} B$. Then $A$ is $\sigma(E, G)$-bounded, $B$ is $\sigma(G, E)$-bounded but not uniformly on $A$, and so is not $\tau(E, G)$-equicontinuous. Hence $\tau(E, G)$ is not barrelled. By Theorem $1, F$ can not have finite codimension in $G$, and so $G=F+M$, where $M \cap F=\{0\}$ and $M$ is of countable dimension.

5. Tweddle and Yeomans [8] investigate the problem of constructing an $H$ for a given barrelled space $E$ such that $\tau(E, F+M)$ is barrelled; they show that this can be done whenever $E$ has a bounded set of dimension greater than or equal to $c$.

De wilde and Schmets $([3], 3)$ consider refining the topology $\tau(E, F)$ by demanding that an extra seminorm $\sup \{|g(x)|: g \in K\}$ be continuous, where $K$ is a convex $\sigma\left(E^{*}, E\right)$-compact subset of $E^{*} \backslash F$. The dual then turns out to be $F+\operatorname{span} K$. However, the proof that the resulting space is barrelled contains an error.' It is not known whether or not

i This was pointed out to us by Dr Tweddle. Professor Schmets writes that he and Professor De :ilde vere aware of this error but have not yet had an opportunity to mention it; they ask that we should do so here. 
$\tau(E, F+\operatorname{span} K)$ is necessarily barrelled. As far as our present paper is concerned, we observe that span $K$ can not be of countable dimension: $\operatorname{span} K=\operatorname{span} A$, where $A$ is the $\sigma\left(E^{*}, E\right)$-closed absolutely convex envelope of $K$ and so is $\sigma\left(E^{*}, E\right)$-compact. Then $\operatorname{span} A$ is a Banach space under the norm topology for which $A$ is the unit ball, and no Banach space can have countable dimension.

6. We turn to the general case, when $M$ is given; then $M=\operatorname{span}\left\{f_{1}, f_{2}, \ldots\right\}$ for some linearly independent sequence $\left(f_{n}\right)$. We write

$$
M^{0}=\{x \in E: f(x)=0 \text { for all } f \in M\}=\bigcap_{n=1}^{\infty} f_{n}^{-1}(0)
$$

thus $M^{0}$ is an intersection of hyperplanes, each dense in $E$ under the barrelled topology $\tau(E, F)$. If $N_{k}=\prod_{n=1}^{k} f_{n}^{-1}(0)$, then $E \supseteq N_{1} \supseteq \ldots \supseteq N_{k} \supseteq N_{k+1} \supseteq \cdots \supseteq M^{0}$. Now $N_{k} \neq N_{k+1}$ for any $k$ (since $\left\{f_{I}, \ldots, f_{k+1}\right\}$ is linearly independent $)$. Hence $M^{0}$ has infinite (countable or uncountable) codimension. The next theorems give information about the topology $\tau(E, F+M)$ in terms of properties of $M^{0}$.

First we need some notation. We write $G^{\sim}$ for the quasicompletion of the vector subspace $G$ of $E^{*}$ under the topology $\sigma\left(E^{*}, E\right) ; G^{\sim}$ is the smallest quasicomplete vector subspace containing $G$. Since a space $E$ is barrelled if and only if its dual is $\sigma\left(E^{*}, E\right)$-quasicomplete, the topology $\tau(E, F+M)$ is barrelled if and only if $F+M=(F+M)^{\sim}$. We have always $F+M \subseteq F+M^{\sim} \subseteq(F+M)^{\sim}$. Now with any locally convex topology is associated a barrelled topology, which we call its kōmura topology. of all barrelled topologies finer than the given topology, it is the coarsest, or alternatively, their inductive limit (Kōmura [5]). Thus $(F+M)^{2}$ is the dual of $E$ under the Kömura topology associated with $\tau(E, F+M)$. We shall use:

LEMMA. Let $E$ and $G$ be Zocally convex spaces, $D$ a dense vector subspace of $E$ barrelled in the relative topology, and $A$ a set of continuous linear mappings of $E$ into $G$. If $A$ is pointwise bounded on 
$D$ then $A$ is equicontinuous (on $E$ ).

Proof. For each closed neighbourhood $V$ of $O$ in $G$, there is an open neighbourhood $U$ of $O$ in $E$ such that $f(D \cap U) \subseteq V$ for all $f \in A$, since $D$ is barrelled and $A$ pointwise bounded. Then $U \subseteq \overline{D \cap U}$ since $D$ is dense; thus $f(U) \subseteq f(\overline{D \cap U}) \subseteq \overline{f(\bar{D} \cap U)} \subseteq \bar{V}=V$ for all $f \in A$.

THEOREM 3. If $M^{0}$ is dense in $E$ and barrelled under the relative $\tau(E, F)$ topology then the Kōmura topology associated with $\tau(E, F+M)$ is $\tau\left(E, F+M^{\sim}\right)$.

Proof. We have to prove $F+M^{\sim}$ quasicomplete. First we show $M^{\sim} \cap E=\{0\}$. If $f \in M^{00} \cap F$ (the second polar being taken in $E^{*}$ ), then $f(x)=0$ on $M^{0}$ and so on $E$, since $M^{0}$ is dense and $f$ continuous. Now $M^{\mathrm{OO}}$ is complete and so $M^{\sim} \subseteq M^{\mathrm{OO}}$; thus $M^{\sim} \cap F=\{0\}$. Let $B$ be a $\sigma\left(F+M^{\sim}, E\right)$-bounded set. Then we can write, uniquely, $g=f+q$ for each $g \in B$, with $f \in F$ and $q \in M^{\sim}$; let $A$ and $Q$ be the sets of such $f$ and $q$ as $g$ runs through $B$. Since $q \in M^{\sim} \subseteq M^{0 O}$, $q(x)=0$ for $x \in M^{0}$, and since $B$ is bounded, $A$ is pointwise bounded on $M^{0}$. By the lemma, $A$ is equicontinuous on $E$ and so lies in an absolutely convex compact subset of $F$. Certainly $A$ is bounded, and so $Q \subseteq B-A$ is a bounded subset of $M^{2}$. Now $M^{\sim}$ is quasicomplete; thus $Q$ lies in an absolutely convex compact subset of $M^{\sim}$. Hence $B$, being a subset of $A+Q$, is $T\left(E, F+M^{2}\right)$-equicontinuous.

NOTE. The lemma is reminiscent of a much used (but not deep) theorem of Banach anł Steinhaus (see, for example, [2], Chapitre V, Théorème 3). If $\left(T_{n}\right)$ is a sequence of continuous linear mappings of a normed space $E$ into a Banach space $F$ such that $\left(T_{n}(x)\right)$ converges on a dense vector subspace $D$ of $E$, and if $\left(\left\|T_{n}\right\|\right)$ is bounded, then $T_{n}(x) \rightarrow T(x)$ on $E$ and $T$ is continuous. One possible generalisation to locally convex spaces $E$ and $F$, with $F$ sequentially complete, is to suppose $D$ barrelled in the place of the hypothesis that $\left(\left\|T{ }_{n}\right\|\right)$ is bounded. The conclusion follows: the lemma shows $\left(T_{n}\right)$ equicontinuous and the rest is 
straight forward.

THEOREM 4. If $M^{O}$ has countable codimension, then $\mathrm{\tau}(E, F+M)$ is not barrezled.

Proof. The relative $\sigma\left(E^{*}, E\right)$ topology on $M^{00}$ is $\sigma\left(M^{00}, E / M^{0}\right)$, since $M^{0}$ is $\sigma\left(E, E^{*}\right)$-closed, and $\operatorname{dim} E / M^{0}=\operatorname{codim} M^{0}$. Hence $M^{00}$ is metrisable, and so also is $M$; therefore $M^{\sim}$ is the completion $M^{00}$ of $M$, and $M \neq M^{\sim}$ since $M$ has countable dimension. Now if $\tau(E, F+M)$ is barrelled, $F+M \subseteq F+M^{\sim} \subseteq(F+M)^{\sim}=F+M$, and so $M^{\sim} \subseteq F+M$. Hence $M^{\sim}=\left(M^{\sim} \cap F\right)+M$ (for $\left.M \subseteq M^{\sim}\right)$. Since $M^{\sim}$ is a Fréchet space and $F$ is quasicomplete, $M^{\sim} \cap F$ is quasicomplete, metrisable, and thus complete, therefore closed; but a Fréchet space can not have a closed vector subspace of countable codimension.

Putting Theorems 3 and 4 together and using Theorem A, we have immediately:

THEOREM 5. If $M^{0}$ is dense and of countable codimension, then $\mathrm{T}(E, F+M)$ is not barrelzed and its associated Kōmura topology is $\tau\left(E, F+M^{00}\right)$.

(The proof of Theorem 4 shows that $M^{2}=M^{00}$ in this case.)

7. The simple construction of Theorem 2 can be refined to show that the hypotheses of Theorem 5 can be satisfied.

THEOREM 6. If $E$ has an infinite dimensional bounded set then there exists $M$ such that $M^{O}$ is dense and of countable codimension in $E$.

Proof. Let $\left\{e_{1}, e_{2}, \ldots\right\}$ be a bounded linearly independent infinite set, and extend to a basis of $E$. Define $f_{n}\left(e_{n}\right)=1$ and $f_{n}$ zero on all other basis elements. Then at most a finite number of the $f_{n}$ are continuous (since the set of $n f_{n}$ is bounded, but not uniformly on the set of $\left.e_{m}\right)$. Discard any that are continuous, put the corresponding $e_{m}$ into the rest of the basis, and relabel (as $f_{1}, f_{2}, \ldots$ and $e_{1}, e_{2}, \ldots$ ). 
Write $M_{1}=\operatorname{span}\left\{f_{1}, f_{2}, \ldots\right\} ;$ then $M_{I}^{0}=\prod_{n=1}^{\infty} f_{n}^{-1}(0)$ and $\operatorname{span}\left\{e_{1}, e_{2}, \ldots\right\}$ is an algebraic supplement of $M_{1}^{0}$. Let $L=\overline{M_{1}^{0}}$. Then $\operatorname{codim} L \leq \operatorname{codim} M_{1}^{0}$ and so $L$ has finite or countable codimension. We may suppose that an algebraic supplement of $L$ has for basis a subset of the $e_{m}$. (Let $u_{1}=e_{i}$ for the least $i$ such that $e_{i} \notin L ;$ let $u_{2}=e_{i}$ for the least $i$ such that $e_{i} \neq \operatorname{span}\left\{L, u_{1}\right\} ;$ if $\left\{u_{1}, \ldots, u_{m}\right\}$ are defined, let $u_{m+1}=e_{i}$ for the least $i$ such that $e_{i} \notin \operatorname{span}\left\{L, u_{1}, \ldots, u_{m}\right\}$. Then each $e_{i}$ is in some $\operatorname{span}\left\{L, u_{1}, \ldots, u_{m}\right\}$ and each $u_{m}$ is some $\left.e_{i}.\right)$

Now $L$ is a closed vector subspace of the barrelled space $E$; if $L$ has countable codimension then $([7], 2, \operatorname{Proposition}) \operatorname{span}\left\{u_{1}, u_{2}, \ldots\right\}$ is isomorphic to the space $\varphi$ (countable direct sum of copies of the scalar field). Hence each bounded set is finite dimensional. But the set of $u_{m}$ is bounded; thus $L$ has finite codimension, $k$ say. If $k=0$, write $M=M_{1}$; if $k>0$, an algebraic supplement of $L$ is spanned by some $\left\{e_{n_{1}}, \ldots, e_{n_{k}}\right\}$. Write $M=\operatorname{span}\left\{f_{n}: n \neq n_{i}, 1 \leq i \leq k\right\}$. Then $e_{n_{i}} \in M^{0} \quad(1 \leq i \leq k)$ and $M_{1}^{0} \subseteq M^{0} ;$ thus $L \subseteq \overline{M^{0}}$ and so $\overline{M^{0}} \supseteq \operatorname{span}\left\{L, e_{n_{1}}, \ldots, e_{n_{k}}\right\}=E$. Hence $M^{0}$ is dense and has countable codimension. Also $M \cap F=\{0\}$, since $M^{0}$ is dense.

NOTES. (1) The proof shows the (curious) fact that if $\left\{e_{1}, e_{2}, \ldots\right\}$ is linearly independent and bounded in a barrelled space, then there exists an algebraic supplement to $\operatorname{span}\left\{e_{m}: m \geq m_{0}\right\}$ for some $m_{0}$ which is dense in $E$.

(2) To each linearly independent bounded sequence $\left(e_{m}\right)$ in $E$ corresponds $M^{0}$, as in the theorem; if $\left(e_{m}\right)$ and $\left(e_{m}^{\prime}\right)$ are two sequences whose spans differ by more than a finite dimensional space, then 
the corresponding $M^{0}$ and $M^{\prime}$ are distinct. Thus in general there are plenty of vector subspaces to which Theorem 5 applies.

8. The codimension of $M^{0}$ is always infinite. Theorem 4 shows that if $\tau(E, F+M)$ is barrelled, then $M^{0}$ has uncountable codimension. Here we show that the converse is not true.

First, we give an example in which $M^{0}$ is dense, barrelled, and of uncountable codimension. This shows that the hypothesis of Theorem 3 may be satisfied even when that of Theorem 4 is not. (There are examples of dense barrelled vector subspaces of uncountable codimension, for instance in [7]; here we elaborate on Saxon and Levin's method to get one of the special form $\prod_{n=1}^{\infty} f_{n}^{-1}(0)$.)

Let $E$ be the product of countably many copies of the reals; then $E$ is a Fréchet space and $S$, the countable (algebraic) direct sum of reals, is dense in $E$. Extend a basis of $S$ to a basis $\left(e_{\alpha}\right)$ of $E$. Since $E$ is c-dimensional, we may suppose that $\alpha$ runs through the interval $(0,1)$, and that the basis elements of $S$ correspond to the values $1-2^{-n}$ of $a(n=1,2, \ldots)$. For each $n$, let $L_{n}=\operatorname{span}\left\{e_{\alpha}: 2^{-n} \leq \alpha<1\right\}$. Then $S \subseteq L_{1},\left(L_{n}\right)$ is increasing, and $E=\bigcup_{n=1}^{\infty} L_{n}$. If each $L_{n}$ is meagre, so is $E$; therefore since $E$ is a Fréchet space, at least one, $L_{k}$ say, is non-meagre. Write $L$ for $L_{k}$. Then $L$ is barrelled, and dense since $S \subseteq L$. Let $L^{\prime}=\operatorname{span}\left\{e_{\alpha}: 0<\alpha<2^{-k}\right\}$. Then $L^{\prime}$ is an algebraic supplement of $L$ and has uncountable dimension.

For each $x$ in $E, x=y+z$, where $y \in L$ and $z \in L^{\prime}$; since $L^{\prime} \subseteq E, z$ is a sequence $\left(\zeta_{n}\right)$. Define $f_{n}(x)=\zeta_{n}$. There must be an infinite subsequence of $f_{n}$ not identically zero. For if $f_{n}=0$ for all $n \geq m, L^{\prime}$ would be m-dimensional. Now $L \subseteq f_{n}^{-1}(0)$ and $L$ is dense, 
so, if $f_{n} \neq 0, f_{n}^{-1}(0)$ is not closed. Hence each such $f_{n}$ is not continuous. Also $L=\prod_{n=1}^{\infty} f_{n}^{-1}(0)$. Put $M=\operatorname{span}\left\{f_{n}: n=1,2, \ldots\right\}$. Then $M^{0}=L$, which has infinite codimension, and so $M$ is not finite dimensional. Thus $M \subseteq E^{*}$, is of countable dimension, and $M \cap F=\{0\}$ since $M^{0}$ is dense; also $M^{0}$ is barrelled and of uncountable codimension.

Now write $E_{1}, F_{1}, M_{1}$ for the $E, F, M$ above; let $E_{2}, F_{2}, M_{2}$ be such that $\tau\left(E_{2}, F_{2}+M_{2}\right)$ is not barrelled, and $M_{2}^{0}$ dense and barrelled (possible by Theorems 6 and 4). Let $E=E_{1} \times E_{2}$. Then $F=F_{1}+F_{2} \subseteq E_{1}^{*}+E_{2}^{*}=E^{*} ;$ let $M=M_{1}+M_{2} \subseteq E^{*}$. Now $\tau(E, E)$ is barrelled, being a product of barrelled topologies. But $\tau(E, F+M)$ is not barrelled, since it is the product of $\tau\left(E_{1}, F_{1}+M_{1}\right)$ and $\tau\left(E_{2}, F_{2}+M_{2}\right)$, and the latter is not barrelled. (If $B_{2}$ is a $\tau\left(E_{2}, F_{2}+M_{2}\right)$-barrel, then $E_{1} \times B_{2}$ is a $\tau(E, E+M)$-barrel.) Also if $N_{1}$ and $N_{2}$ are algebraic supplements of $M_{1}^{0}$ and $M_{2}^{0}$ in $E_{1}$ and $E_{2}$, then $N_{1} \times N_{2}$ is an algebraic supplement of $M^{0}=M_{1}^{0} \times M_{2}^{0}$ in $E_{1} \times E_{2}$. Since $M_{1}^{0}$ has uncountable codimension, so has $M^{0}$. Finally, $M^{0}$ is dense and barrelled, since both $M_{1}^{0}$ and $M_{2}^{0}$ are.

9. Finally, we consider the special case of a normed space.

THEOREM 7. Let $E$ be a normed space. Then $\tau(E, F+N)$ is normable if $N$ has finite dimension; $\tau(E, F+M)$ is metrisable and non-normable if $M$ has countable dimension.

Proof. If $N_{n}=\operatorname{span}\left\{f_{1}, \ldots, f_{n}\right\}$ then $p_{n}(x)=\sup _{1 \leq i \leq n}\left\{\|x\|,\left|f_{i}(x)\right|\right\}$ is a norm on $E$, giving a topology with dual $E+N_{n}$; if $M=\operatorname{span}\left\{f_{1}, \ldots, f_{n}, \ldots\right\}$, the norms $\left(p_{n}\right)$ give a metrisable topology with dual $\bigcup_{n=1}^{\infty}(F+H)=F+M$. 
Now $F$ is a Banach space under the topology $B(F, E)$; if $\tau(E, F+M)$ is normable, $F+M$ is also a Banach space under $B(F+M, E)$ and so is barrelled. Hence $F$, being of countable codimension in $F+M$, is barrelled, by Theorem $A$, under the relative $B(F+M, E)$ topology. But this is coarser than $\beta(F, E)$; hence, by the closed graph theorem, they coincide. Thus $F$ is a complete, and so closed, vector subspace of $F+M$ under $B(F+M, E)$. But then $F$ is a closed vector subspace of countable codimension in a Banach space, which is impossible.

The same argument shows, for example, that if $E$ is a $(D F)$-space under $\tau(E, F)$ then $E$ can not also be a $(D F)$-space under $\tau(E, F+M)$.

\section{References}

[1] Ichiro Amemiya und Yukio Kōmura, "Über nicht-vollständige Montelräume", Math. Arm. 177 (1968), 273-277.

[2] Stefan Banach, Thêorie des opérations linéaires (Monografje Matematyezne, 1. M. Garaskiski, Warszawa, 1932).

[3] M. De Wilde and J. Schmets, "Locally convex topologies strictly finer than a given topology and preserving barrelledness or similar properties", Buzz. Soc. Roy. Sci. Liège 41 (1972), 268-271.

[4] J. Dieudonnê, "Sur les propriétés de permanence de certains espaces vectoriels topologiques", Ann. Soc. Polon. Math. 25 (1952), 50-55 (1953).

[5] Yukio Kōmura, "On linear topological spaces", Kumamoto J. Sci. Ser. A 5 (1962), 148-157.

[6] A.P. Robertson and Wendy Robertson, Topological vector spaces, 2nd edition (Cambridge Tracts in Mathematics and Mathematical Physics, 53. Cambridge University Press, Cambridge, 1973).

[7] Stephen Saxon and Mark Levin, "Every countable-codimensional subspace of a barrelled space is barrelled", Proc. Amer. Math. Soc. 29 (1971), 91-96.

[8] I. Tweddle and F.E. Yeomans, "On the stability of barrelled topologies II", GLasgow Math. J. (to appear). 
[9] Manuel Valdivia, "Absolutely convex sets in barrelled spaces", Am. Inst. Fourier (Grenoble) 21 (1971), fasc. 2, 3-13.

Department of Mathematics, University of Western Australia, Nedlands, Western Australia. 